\title{
Performance Security in Distributed System: Comparative Study
}

\author{
Chliah Hanane \\ IRF-SIC Laboratory \\ Faculty of science, Ibn Zohr \\ University Agadir, Morocco
}

\author{
Amal Battou \\ IRF-SIC Laboratory \\ Faculty of science, Ibn Zohr \\ University Agadir, Morocco
}

\author{
Omar Baz \\ IRF-SIC Laboratory \\ Faculty of science, Ibn Zohr \\ University Agadir, Morocco
}

\begin{abstract}
This paper presents a comparative study of distributed systems and the security issues associated with those systems. Three commonly used distributed systems were considered for detailed analysis in terms of technologies involved, security issues faced by them and solution proposed to circumvent those issues
\end{abstract}

\section{General Terms}

Distributed systems, security

\section{Keywords}

Cloud, Grid and Cluster Computing

\section{INTRODUCTION}

A distributed system in computer science is a bring together system of distributed computers, processors or processes that communicate mutually by common communication medium or network orderly to transmit messages. This distribution could be physical (over a geographical area) or logically (over a virtual space). Coulouris et al., have presented a distributed system as "a system where the hardware and software components have been installed in geographically dispersed computers that coordinate and collaborate their actions by passing messages between them [1]. Tanenbaum and Steen who defined a distributed system as "A collection of independent computers that appears to its users as a single coherent system" [2].

Using a high performance computing (HPC) was limited to community which has financial capacity to extend an exorbitant and very devoted supercomputers of the time. There was a need for small scale HPC, low value which results in computer cluster. The appearance of cluster computing was driven by a varying number of academic project such as Beowulf [3], Berkely NOW [4] and HPVM [5]. Hence, the appeal of the internet and the powerful technologies avaibleness and fast network technologies has modified the way of use. Grid computing was born in academia during 1990s with the purpose to aid users to remotely utilize idle computing capabilities in other centers when local system are busy [3]. Accordingly, after many years of huge concentration in development the grid has grown and come to represent an effective means of coordinated resource sharing and problem solving in dynamic multi-institutional virtual organizations. Cloud computing is a type of computing model that come celebrated at the end of 2007[6]. It provides an ensemble of computing resources that users can access via internet. The concept of cloud resource distribution and allocation take it easier for enterprises to access and use cloud computing resources such as network, storage and data services without having to invest heavily in the infrastructure provided, concentration on other critical activities.

\section{THREE MODELS OF DISTRIBUTED SYSTEM}

\subsection{Cluster computing}

For many years supercomputer was the leader in the field of computing. However enormous issues faced in the area of engineering, business and science which supercomputers failed to deal effectively. Hence cluster computing was replaced by supercomputer with the aim of eliminating these issues and access to potentially huge computing power. Cluster computing is a type of distributed or parallel processing system, which several nodes are combined as a single integrated source [1]. The various nodes implicated in cluster are interconnected by speedy networks such as Myrinet, Ininiband, SCI and Gigabit Ethernet. They work, share and executing compute intensive task as a single computing entity that would be not attainable to execute on a single computer. The clusters are principally used of redundancy, fault tolerant, computing use and load-balancing. They are also used for high availability considering hold redundant nodes that are used to aid service when system components fail. System perform is rectified here because still is one a node ceases, there are different standby nodes that will execute the task and excludes single points of failure without any obstacle [7]. When many computers are boned mutually in a cluster, they share the load of calculational work as a single essential computer. Reasonably, user's outlook signifies they are many computers which functions as an effective machine. The user calculate request are received and distributed each computers in cluster. The consequence of this results in impartial computational duty among different computers, mending the performance of the cluster model. Clusters are recognized to be used frequently in favor of calculational principles, instead than activities based on IO [8].

\subsection{Grid computing}

Grid computing appears in middle 1990 for the objective is to solve large scale computation problem by using the various computing resources of a single cluster [9]. It apply of multiple clusters that are heterogeneous, autonomous, slackly coupled and geographically dispersed [8] as shown in Figure 2 below. Grid computing is consisted as a form of distributed system that involves coordinating and exchanging computing, application, data and storage or network resources across dynamic and geographically dispersed system [10].The innovative idea behind grid environments is to permit the collection of computing resources from many locations to resolve problem that can't be resolve using the processing capability of a single computer. Computers in the grid are not necessary linked in the same physical location and can be operated independently also they can execute of different oss and different Hardware. The motivation of grid computing 
was primarily utilized middleware to among diverse resources( computational and data) across many networks by sharing and allocating pieces of program task among multiple computers as a single virtual system [10].

\subsection{Cloud computing}

Cloud computing is the new pattern based on improvement of virtualization technology which provides large dynamic, scalable pools of virtual IT resources as a service on demand. The concept of cloud computing model is to extend computing, storage and software as a service or as a utility. Depending on Buyya et al. [10] a cloud is a model of parallel and distributed system composed of a collection of interconnected and virtualized computers that are dynamically provisioned and presented as one or more unified computing resource based on service-level agreements (SLA) negotiated, time-honored between consumers and cloud service providers. The infrastructure provide in cloud comprehend scalable resources as network, storage and application that can be rapidly provisioned as well as released with minimal management effort [9].cloud providers essential services that are classified into three levels (Iaas, Paas and Saas)[10].

Software-As-A-Service (SAAS) - This category includes applications that run in a Web browser and do not require any local software and hardware besides the Web browser and Internet connection. Examples of software in this category are Google Docs [11] and Microsoft Office 365 [12]

Infrastructure-As-A-Service (IAAS) - This category includes virtualization software that allows an operating system to be run in the cloud. Typically, the user will pick a hardware configuration and install an operating system into the virtual hardware configuration. IAAS was designed to free the user from the purchase of hardware and allow for hardware upgrades easily known also as Hardware as a Service (Haas). Examples of IAAS offerings are Amazon EC2 [13] and Rackspace [14].

It can be divided into several categories, the most important being:

(a) Computing as a Service (Caas) where virtual machines are rented and priced according to resource consumption in a given unit of time, the main memory, CPU, features of its operating system and pre-installed applications.

(b) Data as a Service (Daas), also known as storage as a Service (staas), where virtually unlimited storage space is provided for storing files, regardless of size and type, being charged according to the amount of data stored or transferred.

(c) Network as a Service (Network as a Service - Naas) refers to cloud services which provide virtual network connections, such as VPN or MVNO, or by infrastructure sharing that may belong to third parties.

Platform-As-A-Service (PAAS) - This category includes prebuild components that a developer can use when developing a cloud application. The goal of PAAS is to allow the developer to focus on the development of a solution for the business functions and not software functions that span many application domains. A good example of PAAS is force.com where the developer is provided many of the essential parts of an application out of the box.

Recent scientific literature presented novel concepts of different applications offered as a services (xaas), culminating in the highest level in the form of people offered as a service on the pile of basic cloud computing, which is called human as a services (huaas). The motivation of this approach is emphasizes that the paradigm of cloud computing can be large to afford services across the participation of human beings as a resources [15].

\section{ADVANTAGES AND DISADVANTAGES}

\subsection{Cluster Computing}

The fundamental features of cluster computing illuminate of the following [16]

Single system image

Centralized Job management and planning system

Tightly coupled systems

Table 1. Table advantages and disadvantages of Cluster Computing

\begin{tabular}{|c|c|}
\hline Advantages & Disadvantages \\
\hline High availability & $\begin{array}{c}\text { Difficult for developing } \\
\text { software for distributed system }\end{array}$ \\
\hline Single Systele Image & Difficult for hand by a Layman \\
\hline Improves network technology & $\begin{array}{c}\text { Easily accessed and applied to } \\
\text { secret data }\end{array}$ \\
\hline Reducing cost & Programmability issues \\
\hline Manageability & Problem in finding fault \\
\hline
\end{tabular}

\subsection{Grid Computing}

Grid computing is employing the resources of several computers in a network to a single problem at the same time generally to a scientific as well as technical problem that requires a great number of computer processing cycles [17].

The essential characteristics of grid can be detailed as follows [6], [8]:

Resource sharing

Geographical distribution

Heterogeneity

Large scale

Multiple administrations

Resource coordination

Transparent access

Dependable access

Consistent access

Pervasive access

Decentralization (Loosely coupled)

Dynamism and Diversity

Distributed Job Management \& scheduling

Table 2. Table advantages and disadvantages of Grid Computing

\begin{tabular}{|c|c|}
\hline Advantages & Disadvantages \\
\hline Reliability & $\begin{array}{c}\text { High Internet connetion } \\
\text { required }\end{array}$ \\
\hline
\end{tabular}




\begin{tabular}{|c|c|}
\hline $\begin{array}{c}\text { Acces to additional } \\
\text { ressources }\end{array}$ & $\begin{array}{c}\text { Diffrent administrator } \\
\text { domains }\end{array}$ \\
\hline $\begin{array}{c}\text { Computers working } \\
\text { together }\end{array}$ & $\begin{array}{c}\text { Technology support to } \\
\text { utilize the grid }\end{array}$ \\
\hline $\begin{array}{c}\text { Can solve larger, more } \\
\text { complex problems in a } \\
\text { shorter time }\end{array}$ & $\begin{array}{c}\text { Grid software and standars } \\
\text { are still evolving }\end{array}$ \\
\hline Ressource balancing & Not stable \\
\hline $\begin{array}{c}\text { Unsed computing capacity } \\
\text { is effectively used }\end{array}$ & $\begin{array}{c}\text { Some applications cannot } \\
\text { be parallezed }\end{array}$ \\
\hline $\begin{array}{c}\text { Make better use of existing } \\
\text { hardware }\end{array}$ & $\begin{array}{c}\text { Learning curve to get } \\
\text { started }\end{array}$ \\
\hline $\begin{array}{c}\text { Easier to collaborate with } \\
\text { other organizations }\end{array}$ & $\begin{array}{c}\text { Non-interactive job } \\
\text { submission }\end{array}$ \\
\hline
\end{tabular}

\subsection{Cloud Computing}

A current computing paradigm are Cloud Computing [18] that providing IT infrastructure and fundamental Services i.e. Infrastructure as a service (iaas), platform as a service (paas), software as a service (saas) etc.

Cloud computing [17] is a weighty model to empower ubiquitous, accessible, on demand network access To a shared pool of configurable computing resources as networks, servers, storage, applications, and Services that can be speedily provisioned as well as liberated with minimal management effort.

The fundamental characteristics of cloud computing can be elaborated as follows [8]:

On-demand self-service

Broad network access

Resource pooling

Rapid elasticity

Measured service

Table 3. Table advantages and disadvantages of Cloud Computing

\begin{tabular}{|c|c|}
\hline Advantages & Disadvantages \\
\hline $\begin{array}{c}\text { Automatic Software } \\
\text { Integration }\end{array}$ & $\begin{array}{c}\text { Dependency and } \\
\text { Vendor lock-in }\end{array}$ \\
\hline Versatile Compatibility & No always many room \\
\hline Almost Unlimited Storage & Security in the Cloud \\
\hline $\begin{array}{c}\text { Better Hardware } \\
\text { Management }\end{array}$ & $\begin{array}{c}\text { Increased } \\
\text { Vulnerability }\end{array}$ \\
\hline $\begin{array}{c}\text { Shared Resources } \\
\text { Easy Access to } \\
\text { Information }\end{array}$ & Internet Required \\
\hline Cost Efficient & Non-Interoperability \\
\hline Mobility & Less Control \\
\hline Quick Deployment & Technical Issues \\
\hline
\end{tabular}

\section{SECURITY IN DISTRIBUTED}

\section{SYSTEM}

\subsection{Cluster Computing Security}

The security performance problem becomes more complicated as cluster systems use very high speed cluster interconnects. Traditionally, all communication tasks such as packetizing and interrupts handling are done by the operating system, consuming a host CPU's computing power. As cluster interconnects become faster. The communication-handling overhead becomes more pronounced. M. Lee, E. J. Kim, K. H. Yum, and M. Yousif, addresses security issues in cluster interconnect, especially with user-level communication (ULC), compare possible schemes to enable ULC security; host processor, secure coprocessor, and security enforced network interface card (SENIC) and strongly advocated SENIC for its low end-to-end latency and high security transparency while retaining the performance benefit of ULC through by passing the host processor [19].

Bourzandi and Gordon present the security approach of corresponding the operator-class cluster environment is an independent security model that includes distributed authentication and distributed access control. The security approach to HPC cluster environment focuses on multidimensional detection designed to enable real-time security management of clusters that adapts quickly and automatically to changing situations. Nvisioncc is the first security intrusion detection system specifically designed for the unique HPC cluster environment [20].

$\mathrm{Li}$ and Qin have presented the security of computing clusters using exploitation graphs (e-graphs). They have put in place different attacks that can be used on all three model of security such as confidentiality, availability and integrity, also demonstrate that this technique could be used to certify clusters using a Cluster Vulnerability Knowledge Database [21].

Thalor and Niwas have proposed a security model for computer networks based on cluster computing architecture, by employing various tools available in the TCP / IP security model. Each tool has its own security features that make the system secure, have used these security tools with their security functions at different levels of cluster computing architecture, to form a secure high-performance computing system [22].

As a result, it can be seen that clusters of computers are vulnerable to attack by malevolent elements such as hackers and crackers owing of their open nature and the use of public resources such as the Internet. Considerable research has been conducted by several researchers on cluster security and has proposed several methods that can be used to protect clusters from these attacks.

\subsection{Grid Computing Security}

The security has been a central issue in grid computing born the outset, and has been regarded as the most significant challenge for grid computing. A. Bendahmane, M. Essaaidi, A. El Moussaoui, A.Younes are proposed the classification of the different mechanisms and solutions pertaining to the different components of grid computing security. This classification subdivide the grid computing security into five main categories, which are Resources Level, Service Level, Authentication \& Authorization Level, Information Level, and Management Level Solutions [23].

Particularly are Grid computing sensitive to authentication and authorization due to its decentralized type. Authentication in a grid computing environment is the first line of defense before any other security aspects [24]. It treats with the verification of the identity of an entity within a network. [25]. Has categorized authentication methods into: kerberos authentication, password authentication, secure sockets layer (SSL) authentication, and certification authorities (CA). The authorization is one the process that define whether a particular operation is authorized. In grid computing, 
authorization systems can be splitted into two categories: systems of level VO (virtual organization) have a centralized authorization system that affords credentials to users to access resources and systems of level of resources are decentralized and permit users to access resources based on the credentials presented by users [25].

\subsection{Cloud Computing Security}

Cloud computing offers scalable on-demand services to users with greater flexibility. Since cloud services are delivered using classical network protocols, implicit vulnerabilities, as well as, network threats raise more security and privacy concerns [26]. [27] surveyed the research on cloud security issues, addresse several key topics such as: vulnerabilities, threats, and attacks. Focused on the so-called SPI model (saas, paas and iaas); another categorization was done.

[26] classified security concerns based on different layers of the cloud infrastructure as: Application Level, Network Level, Data storage Level, Virtualization Level, Authentication and access control Level, Trust Level, Compliance, audit and regulations Level. Application level risks directly affect the security of cloud applications at the user layer. Network level threats or intrusions affect the overall security of cloud services, data as well as physical resources. Since one can easily gain access to another user's resources or services by monitoring the network traffic in the cloud. Attacks on data storage directly affect the security of the user's data (at rest or in-transit) including application data and sensitive data. Virtualization level risks directly affect the data storage and application security levels. Authentication and access control level risks affect the security of authentic user's services and resources.

\section{CONCLUSION}

In this paper, attempted the best to discuss a detailed comparison of the three models of computation, cluster, grid and cloud computing. The notion of cloud computing is becoming more and more popular and it is in the beginning level. Accepted companies are furnishing all types of cloud computing service, from software application to net storage and will suppose cloud computing will become principal technology in information life. Grid, cloud and more computing seems to be promising model, particularly focused on api standardization, security, interoperability, new business models, as well as dynamic pricing systems for complex services. Making a security model based on data encryption algorithm will be future research.

\section{REFERENCES}

[1] G. F. Coulouris, Ed., 2012, Distributed systems: concepts and design, 5th ed. Boston: Addison-Wesley.

[2] A. S. Tanenbaum and M. van Steen, 2007, Distributed systems: principles and paradigms, 2nd ed. Upper Saddle RIiver, NJ: Pearson Prentice Hall.

[3] "Beowulf.org | Mailing List and Archives." [Online]. Available: http://www.beowulf.org/. [Accessed: 30-Jun2017].

[4] "High Performance Virtual Machines." [Online]. Available:

http://cseweb.ucsd.edu/groups/csag/html/projects/hpvm.h tml. [Accessed: 30-Jun-2017].

[5] T. E. Anderson, D. E. Culler, D. A. Patterson, and and the N. team, "A Case for NOW (Networks of Workstations)," IEEE Micro, vol. 15, no. 1, pp. 54-64, Feb. 1995.

[6] N. Sadashiv and S. D. Kumar, "Cluster, grid and cloud computing: A detailed comparison," in Computer Science \& Education (ICCSE), 2011 6th International Conference on, 2011, pp. 477-482.

[7] P. Purnawansyah and R. Sastra, 2016, "Cluster Computing Analysis Based on Beowulf Architecture,' Int. J. Comput. Inform. IJCANDI, vol. 1, no. 1, pp. 9-16.

[8] "A Comparative Analysis- Grid, Cluster and Cloud Computing.pdf." .

[9] I. Foster, Y. Zhao, I. Raicu, and S. Lu, 2008, "Cloud Computing and Grid Computing 360-Degree Compared,", pp. 1-10.

[10] R. Buyya, C. S. Yeo, S. Venugopal, J. Broberg, and I. Brandic, 2009, "Cloud computing and emerging IT platforms: Vision, hype, and reality for delivering computing as the 5th utility," Future Gener. Comput. Syst., vol. 25, no. 6, pp. 599-616,( Jun. 2009).

[11] "Google Docs - create and edit documents online, for free." [Online]. Available: https://www.google.com/docs/about/. [Accessed: 30-Jun2017].

[12] "Office Products - Microsoft Office." [Online]. Available: https://products.office.com/enus/products?tab=O-Home. [Accessed: 30-Jun-2017].

[13] "Elastic Compute Cloud (EC2) - Cloud Server \& Hosting - AWS," Amazon Web Services, Inc. [Online]. Available: //aws.amazon.com/ec2/. [Accessed: 30-Jun2017].

[14] "Rackspace: Managed Dedicated \& Cloud Computing Services," Rackspace Hosting. [Online]. Available: https://www.rackspace.com. [Accessed: 30-Jun-2017].

[15] "A Comparative Study of SaaS, PaaS and IaaS in Cloud.pdf.".

[16] "Office Products - Microsoft Office." [Online] Available: https://products.office.com/enus/products?tab=O-

Homehttp://www.tutorialspoint.com/cloud_computing/. [Accessed: 30-Jun-2017].

[17] H. AlHakami, 2012, "Comparison Between Cloud and Grid Computing: Review Paper," Int. J. Cloud Comput. Serv. Archit., vol. 2, no. 4, pp. 1-21, (Aug. 2012).

[18] M. L. Bote-Lorenzo, Y. A. Dimitriadis, and E. GómezSánchez, 2004, "Grid Characteristics and Uses: A Grid Definition," in Grid Computing, vol. 2970, F. Fernández Rivera, M. Bubak, A. Gómez Tato, and R. Doallo, Eds. Berlin, Heidelberg: Springer Berlin Heidelberg, pp. 291-298.

[19] M. Lee, E. J. Kim, K. H. Yum, and M. Yousif, 2006, “An overview of security issues in cluster interconnects," in Cluster Computing and the Grid, 2006. CCGRID 06. Sixth IEEE International Symposium on, vol. 2, p. 9-pp.

[20] M. Pourzandi, D. Gordon, W. Yurcik, and G. A. Koenig, "Clusters and security: distributed security for distributed systems," in Cluster Computing and the Grid, 2005. IEEE International Symposium on, 2005, vol. 1, pp. 96104.

[21] Wei Li and R. B. Vaughn, 2006,"Cluster Security Research Involving the Modeling of Network Exploitations Using Exploitation Graphs,” pp. 26-26.

[22] S. K. Thalod and R. Niwas, 2013, "SECURITY MODEL FOR COMPUTER NETWORK BASED ON CLUSTER COMPUTING," Int. J. Eng. Comput. Sci. ISSN, pp. 1920-1927. 
[23] "Grid Computing Security Mechanisms the state-of-theart.ppt.".

[24] A. Farouk, A. A. Abdelhafez, and M. M. Fouad, 2012, "Authentication mechanisms in grid computing environment: Comparative study," in Engineering and Technology (ICET), 2012 International Conference on, pp. 1-6.

[25] R. Bhatia, "Grid Computing and Security Isses, 2013,"
Int. J. Sci. Res. Publ., vol. 3.

[26] C. Modi, D. Patel, B. Borisaniya, A. Patel, and M. Rajarajan, 2013, "A survey on security issues and solutions at different layers of Cloud computing," J. Supercomput., vol. 63, no. 2, pp. 561-592, Feb.

[27] A. Diogo et al., 2014, "Security issues in cloud environments: a survey,” Int. J. Inf. Secur., vol. 13, no. 2, p. 113. 